\title{
Casos de dengue e coleta de lixo urbano: um estudo na Cidade do Recife, Brasil
}

\author{
Cases of dengue and urban waste collection: \\ a study in the City of Recife
}

Marcos Felipe Falcão Sobral ${ }^{1}$

Ana Iza Gomes da Penha Sobral ${ }^{2}$

${ }^{1}$ Departamento de Administração, Universidade Federal Rural de Pernambuco. Rua Dom Manoel de Medeiros s/n, Dois Irmãos. 55002-970 Recife PE Brasil. mffsobral@gmail.com ${ }^{2}$ Universidade Federal de Pernambuco. Recife PE Brasil.

\begin{abstract}
The scope of this study was to identify which categories of urban waste are associated with cases of dengue and to evaluate the impact of garbage collection on dengue infection in the City of Recife (Brazil). Data from categorized waste weighing and the confirmed cases of dengue in the city were used. The data were analyzed using Pearson's correlation coefficient for the 13 categories of urban garbage, followed by Multivariate Linear Regression, selecting the variables by the stepwise method. A negative correlation between dengue infections in seven categories was identified: household garbage $(r=-0.835)$, differentiated residues $(r=-0.835)$, special operations residues ( $r=-0.711)$, building rubble ( $r=$ $-0.687)$, selective waste collection $(r=-0.425)$ and tires $(r=-0.423)$. The regression model was able to explain $75 \%$ of the variation, indicating that an increase of 1,000 tons in household garbage collection provides a decrease of 0.032 in cases of dengue, while the same increase in tire collection esults in a decrease of 0.465 . The results show that garbage collection has a strong negative impact on dengue cases and can be adopted as a prevention strategy by municipal governments.
\end{abstract}

Key words Dengue, Garbage collection, Aedes aegypti
Resumo O objetivo desse estudo foi identificar quais categorias de lixo urbano apresentam associação com casos de dengue e, em seguida, avaliar o impacto da coleta de lixo sobre os casos da doença na cidade do Recife. Foram utilizados dados da pesagem categorizada de lixo, juntamente com os casos confirmados de dengue no município. Os dados foram analisados através do coeficiente de correlação de Pearson para as treze categorias de lixo, seguido pela Regressão Linear Multivariada, selecionando as variáveis pelo método de "stepwise". Identificou-se a existência de correlação negativa entre o total de casos de dengue em sete categorias: lixo domiciliar $(r=-0,835)$, resíduos diferenciados $(r=-0,835)$, resíduos de operações especiais $(r=-0,711)$, entulhos $(r=-0,687)$, coleta seletiva $(r=-0,425)$ e pneus $(r=-0,423)$. O modelo de regressão foi capaz de explicar $75 \%$ da variação, apontando que um incremento de 1.000 toneladas na coleta de lixo doméstico proporciona uma redução de 0,032 casos de dengue enquanto que o mesmo incremento na coleta de pneus é capaz de reduzir 0,465 casos da doença. Os resultados demonstram que a coleta de lixo possui um forte impacto negativo nos casos de dengue e podem ser adotados como estratégia de prevenção pelos governos municipais.

Palavras-chave Dengue, Coleta de lixo, Aedes aegypti 


\section{Introdução}

A dengue é a mais importante infecção viral transmitida por artrópodes aos humanos, colocando em risco aproximadamente 2,5 bilhões de pessoas em todo o mundo ${ }^{1}$. O vírus da dengue é um membro da família dos flavivírus, com outros membros causadores da Febre do Nilo Ocidental, Zika, Febre Amarela e Chikungunya ${ }^{2}$. Geralmente, a dengue incide com mais frequência em áreas tropicais, sendo as epidemias comumente oriundas no verão, durante ou após períodos de chuvas $^{3}$, isso porque a água se acumula em calhas, pneus abandonados e fossas sépticas, fornecendo criadouros convenientes para reprodução dos mosquitos ${ }^{4}$. Dessa forma, a eliminação de criadouros propícios à reprodução de seu vetor, o Aedes aegypti, é uma das mais importantes formas de prevenir casos de dengue e das demais arboviroses transmitidas pelo mosquito ${ }^{5-8}$.

Apesar das ações de prevenção, os esforços de controle, notadamente, não conseguiram travar a crescente incidência de epidemias de dengue e a expansão da distribuição geográfica de transmissão endêmica'. Fatores como níveis elevados de precipitação, temperatura, proximidades de periferias urbanas e baixa renda estão associados a um maior risco de dengue 9 .

De acordo com a Organização Mundial da Saúde $(\mathrm{OMS})^{10}$ estima-se que, anualmente, ocorrem 50 milhões de infecções de dengue no mundo. O Brasil, por ser um país tropical e apresentar condições que favorecem o desenvolvimento e a proliferação do principal transmissor da doença, passou por um período de epidemia recentemente, tendo em 2013 o maior número de casos notificados, cerca de 2 milhões de casos $^{11}$.

Além de aspectos climáticos e topográficos, já foram identificados diversos fatores sociais que também podem estar associados ao aumento do Aedes aegypti, dentre eles, a coleta de lixo. Em estudo realizado em Rajasthan, na Índia, verificou-se que o aumento na frequência da coleta de lixo, interfere na reprodução do mosquito, reduzindo assim a transmissão de doenças ${ }^{11}$. $O$ estudo também apontou que a manutenção de resíduos sólidos, por períodos superiores a sete dias, apoia a criação do mosquito. Em contrapartida, a intervenção adequada na gestão de resíduos sólidos, via programas de prevenção, foi capaz de reduzir o número de pupas nas comunidades que receberam uma coleta de lixo mais frequente e melhor organizada ${ }^{12}$.

A coleta de lixo urbana é uma das principais medidas sanitárias que ajuda a prevenir a pro- liferação de doenças em zonas urbanas e rurais. A presença humana em uma região é capaz de produzir um alto sortimento e volume de resíduos $^{13}$, que precisam ser coletados e devidamente tratados. No ano de 2014, o Brasil gerou em média $0,963 \mathrm{~kg} /$ habitante/dia de resíduos enquanto que a Região Nordeste gerou 0,771 kg/habitante/ dia, sendo essa $2,8 \%$ maior que $2013^{14}$. Os municípios da Região Nordeste aplicaram em média $\mathrm{R} \$ 8,37 /$ habitante/mês para realização de todos os serviços relacionados com limpeza urbana e coleta de resíduos sólidos, sendo esse valor abaixo da média nacional de $\mathrm{R} \$$ 9,98/habitante/mês ${ }^{14}$.

O poder público é o responsável pelo funcionamento dos serviços de limpeza urbana, sendo que em Recife a coleta e limpeza são executadas em nível municipal, através de concorrências pri$\operatorname{vadas}^{15}$. Os resíduos coletados no município são pesados antes do depósito em aterros sanitários, permitindo a elaboração de uma análise entre as pesagens e os casos de dengue na região. Dessa forma, o presente estudo tem como finalidade identificar quais são as categorias de lixo que apresentam associação com a dengue, avaliando em seguida o impacto do volume de lixo coletado (em toneladas) sobre os casos confirmados da doença na cidade do Recife.

\section{Métodos}

Trata-se de um estudo quantitativo, transversal e analítico, onde foram utilizados dados secundários do Portal de Dados Abertos da Cidade do Recife, referentes ao período de janeiro de 2013 a fevereiro 2015 , totalizando 26 meses, sendo as informações de acesso público, sem a identificação dos usuários e/ou vítimas. Esse estudo não utilizou amostra e tomou como base os dados oriundos do universo total.

Foram analisados dois grupos de variáveis. O primeiro, relativo ao número de casos confirmados de dengue (CID-10 A90) em todos os distritos sanitários do Recife durante o período mencionado, sendo considerados os registros no qual a classificação final estava assinalada como Dengue Clássico, Dengue com Complicações, Febre Hemorrágica do Dengue, Dengue, Dengue com Sinais de Alarme e Dengue Grave. Todas as ocorrências registradas na base de dados foram confirmadas através de exame laboratorial ou clínico epidemiológico.

$\mathrm{O}$ segundo grupo de dados, relativos à pesagem de lixo urbano, foram medidos em toneladas/mês e categorizados em 13 grupos: domici- 
liar, canais/galerias/raspagens, coleta seletiva, entulhos, resíduos diferenciados, resíduos de operações especiais, podação/compostagem, pneus, asfalto, plástico, metal, papel e coletas em pontos críticos. Os dados foram oriundos das medições da prefeitura. Para medir o volume de lixo o veículo é pesado totalmente carregado, quando entra no aterro e, em seguida, é pesado descarregado, na saída do aterro. Essa diferença é considerada como carga de lixo. Como as empresas são remuneradas pelo volume de lixo coletado, existem fiscais da Prefeitura da Cidade do Recife, acompanhando o processo.

Para o tratamento estatístico foi aplicado o coeficiente de Correlação de Pearson entre as 13 categorias de resíduos e a ocorrência dos casos de dengue. Além disso, foi calculada a regressão linear multivariada, com modelo de regressão stepwise. O nível de significância foi de 5\%, enquanto que o intervalo de confiança foi de $95 \%$. Os dados foram organizados e tratados em planilhas do Excel. Para a análise estatística foi utilizado o Software IBM SPSS Statistics 22. Para fins estatísticos, foi considerado o mês da notificação dos casos e o mês da pesagem do resíduo.

\section{Resultados}

Durante o período estudado, foram confirmados 3.972 casos de Dengue na cidade do Recife, com $\mu=152,8 /$ casos/mês e $=323,3$. Do total de casos, 2.264 (57\%) eram mulheres, 1.706 (43\%) homens e dois $(0,1 \%)$ não informaram sexo. Quanto à forma de diagnóstico, 583 casos foram confirmados via exames laboratoriais, enquanto que 3.389 foram confirmados através de exames clínicos.

As pesagens de lixo do período totalizaram 2.153.843 toneladas, que foram classificadas pela Prefeitura do Recife em doméstico (53,07\%), entulhos e metralhas (24,14\%), raspagem (16,01\%), resíduos de aplicação asfáltica $(2,73 \%)$, resíduos de operações especiais $(2,47 \%)$, resíduos diferenciados $(1,20 \%)$, coleta seletiva $(0,23 \%)$, podação de árvores e compostagem $(0,07 \%)$, pneus $(0,03 \%)$, limpeza de pontos críticos $(0,02 \%)$, plástico $(0,01 \%)$, papel $(0,01 \%)$ e metal $(0,01 \%)$.

\section{Correlação}

Todas as correlações estatisticamente significativas foram negativas, variando de fraca a forte. Foi encontrada uma forte correlação negativa entre o total de casos confirmados de dengue e a pesagem mensal não categorizada de lixo com $r$ $=-0,700$ e $\mathrm{P}<0,001$.

Considerando as pesagens categorizadas de lixo, foi identificada uma forte correlação negativa entre coleta de lixo domiciliar $(-0,835)$ e casos de dengue. Além de apresentar a associação mais forte entre os casos de dengue, a coleta domiciliar também representou a maior fração da pesagem de lixo do período.

A coleta de lixo diferenciado está relacionada com ações da prefeitura para recolhimento de resíduos fora de padrão, tais como refrigeradores e caixotes. Essa categoria também apresentou uma forte correlação negativa com os casos de Dengue $(-0,809)$. Já os resíduos de operações especiais, tratam de mutirões e campanhas sazonais para redução dos rejeitos em determinadas regiões e apresentou correlação negativa moderada $(-0,711)$.

O recolhimento de entulhos, metradas e resíduos de construção civil também apresentaram associação moderada e negativa $(-0,687)$, seguido por coleta seletiva $(-0,425)$ e pneus $(-423)$, que indicaram associação fraca e negativa. A raspagem de canais e galerias, atividades de podação de árvores e compostagem, resíduos de aplicação asfáltica, plástico, papel e metal não apresentaram associações ao nível de significância $=0,05$. A Tabela 1 indica as associações estatisticamente significativas que foram apuradas.

\section{Regressão}

A regressão stepwise excluiu 11 variáveis, por apresentarem p-valores acima no nível de significância de 5\%. Resultaram do modelo duas variáveis significantes, sendo a primeira a coleta de lixo doméstico e a segunda a coleta de pneus.

Tabela 1. Correlações Coleta de Lixo e Casos Confirmados de Dengue.

\begin{tabular}{lcc}
\hline \multicolumn{1}{c}{ Variável } & $\begin{array}{c}\text { Correlação } \\
\text { de Pearson }\end{array}$ & $\begin{array}{c}\text { Significância } \\
(2 \text { extremidades })\end{array}$ \\
\hline $\begin{array}{l}\text { Coleta total de lixo (não } \\
\text { categorizada) }\end{array}$ &,- 700 & $\mathrm{P}<0,001$ \\
Domiciliar &,- 835 & $\mathrm{P}<0,001$ \\
Resíduos Diferenciados &,- 809 & $\mathrm{P}<0,001$ \\
Resíduos Operações &,- 711 & $\mathrm{P}<0,001$ \\
Especiais & & \\
Entulhos &,- 687 & $\mathrm{P}<0,001$ \\
Coleta Seletiva &,- 425 & $\mathrm{P}=0,030$ \\
Pneus &,- 423 & $\mathrm{P}=0,032$ \\
\hline
\end{tabular}


O R-Quadrado foi de 0,751 , o que significa que o modelo com as variáveis "Lixo Domiciliar" e "Pneus", é capaz de explicar até $75,1 \%$ da variação nos casos de dengue. A ANOVA, com total de 25 graus de liberdade apresentou significância de $\mathrm{P}<0,001$, o que reforça a afirmação que ao menos uma variável está influenciando nos casos de Dengue.

Foi confirmada a ausência de multicolinearidade, ou seja, não existe correlação entre as variáveis preditoras. No modelo, a tolerância apurada foi de 0,945 , sendo recomendável acima de 0,1, enquanto que o Variance Inflation Factor foi de 1,059, sendo esse abaixo de 10. De acordo com o Teste KS (Komogorov-Smirnov), os resíduos estão distribuídos de forma normal, com p-valor de 0,042 ao nível de significância de 0,05.

O coeficiente escalar encontrado foi de 1714,92 , enquanto que o preditor Domiciliar apresentou valor de B de -0,0000324939761601456, com significância de 0,000 , e o preditor Pneus valor de B de -0,00464469675863561, com significância de 0,036 . O preditor que mais influencia a variável dependente é a coleta de lixo domiciliar, apresentando um coeficiente padronizado Beta de $-0,779$, seguido por $-0,239$ da coleta de pneus. Os dados estão dispostos na Tabela 2.

O procedimento stepwise excluiu onze variáveis por não atenderem ao nível de significância, sendo essas: Canais/Galerias/Raspagens, Coleta Seletiva, Entulhos, Resíduos Diferenciados, Operações Especiais, Podação/Compostagem, Asfalto, Plástico, Metal, Papel e Pontos Críticos.

\section{Discussão}

As diversas esferas do poder público têm direcionado esforços em campanhas de conscientização da população no sentido de reduzir criadouros de mosquitos, ações como, por exemplo, a eliminação de água parada. Porém, o controle de veto-

Tabela 2. Modelo final da regressão linear múltipla entre características da coleta do lixo urbano e casos de dengue em Recife, 2013-2015.

\begin{tabular}{lrrrrr}
\hline \multirow{2}{*}{ Variável } & \multicolumn{2}{c}{$\begin{array}{c}\text { Coeficientes não } \\
\text { padronizados }\end{array}$} & $\begin{array}{c}\text { Coeficientes } \\
\text { padronizados }\end{array}$ & t & Significância \\
\cline { 2 - 4 } & B & $\begin{array}{c}\text { Erro } \\
\text { Padrão }\end{array}$ & Beta & & \\
\hline Constante & 1714,923 & 194,243 & & 8,829 & $\mathrm{P}<0,001$ \\
Domiciliar & $-3,249 \mathrm{E}-5$ &, 000 &,- 779 & $-7,271$ & $\mathrm{P}<0,001$ \\
Pneus &,- 005 &, 002 &,- 239 & $-2,234$ & $\mathrm{P}=0,036$ \\
\hline
\end{tabular}

res e os programas de prevenção são, frequentemente, insuficientes, ineficazes ou ambos ${ }^{1}$. Isso se deve em parte porque o vetor da dengue, o Aedes aegypti, apresenta uma grande capacidade de adaptação, podendo se reproduzir inclusive em águas poluídas e em altitudes elevadas ${ }^{16}$.

Os resultados desta pesquisa apontam para uma direção já há muito conhecida, porém, pouco explorada nas ações de prevenção, em que a coleta regular de resíduos e a redução do lixo doméstico pelo poder público podem trazer significativos ganhos na redução populacional do Aedes aegypti ${ }^{17-19}$.

Estudos já evidenciaram que existe um grande volume de criadouros positivos provenientes do lixo doméstico ${ }^{20}$, o que gera a necessidade de atenção sobre a importância do correto acondicionamento dos resíduos ${ }^{18}$. Porém, tão importante quanto acondicionar corretamente o lixo, está a coleta eficiente e regular, uma vez que se relaciona diretamente com o controle do Aedes aegyptit ${ }^{21}$.

Os dados dessa pesquisa sugerem que existe correlação negativa estatisticamente significante entre a coleta de lixo e a incidência do dengue na cidade do Recife. Esse resultado é corroborado em estudos realizados em Quenzon City ${ }^{22}$ e São José do Rio Preto ${ }^{23}$ onde os casos de dengue também estão correlacionados negativamente com o volume de lixo ou frequência na coleta. Já em pesquisa realizada no Espírito Santo, as co-variáveis que melhor explicaram o risco de dengue foram coleta ineficiente do lixo e renda mensal inferior a três salários mínimos ${ }^{24}$.

Embora, por si só, não seja possível estabelecer uma relação de causalidade, verifica-se que, à medida que as pesagens de lixo aumentam, existe uma redução na incidência de casos de Dengue. Essa correlação, em Recife, é bem mais acentuada quando se trata das categorias lixo doméstico e resíduos diferenciados.

Em se tratando de forma específica à coleta seletiva, apesar de essa já ter sido proposta como um indicador de vigilância da dengue ${ }^{25}$, os dados do modelo para a cidade do Recife apresentaram uma correlação fraca e negativa para essa categoria de resíduos. A principal causa está ligada ao volume apurado, apenas $0,23 \%$ do total de pesagens. Cabe destacar que a ausência de coleta seletiva ocasiona grandes volumes de lixo doméstico, uma vez que o descarte é feito de modo genérico e não categorizado.

É sabido que os recipientes provenientes de lixo doméstico, por possuírem um menor tamanho, podem proporcionar um rápido acúmulo 
de água, especialmente durante o verão ${ }^{20}$. Dessa forma, frequência e volume da coleta de lixo podem influenciar na variação populacional do mosquito e consequentemente, no volume de pessoas infectadas pelos arbovírus ${ }^{26,27}$.

$\mathrm{Na}$ cidade do Recife, a frequência de coleta de lixo pode ocorrer diariamente ou três vezes na seman $a^{28}$, sendo definida em âmbito municipal e executada por empresas terceirizadas. Usualmente, a frequência é determinada pela capacidade de uma região ou rota de atingir a capacidade máxima do veículo, na faixa econômica de operação do veículo coletor. Segundo o modelo, o incremento no volume coletado diariamente pode trazer importantes ganhos no combate à doença, mesmo que isso implique aumento de frequência de coletas ou em rotas com volume inferior à faixa ótima de operação dos caminhões.

Se considerarmos apenas os preditores estatisticamente significativos pela regressão, é possível afirmar que para cada mil toneladas de lixo doméstico coletado a mais, tem-se uma redução de 0,032 casos de dengue. Quando se analisa a coleta de pneus, observa-se um potencial bem mais elevado, sendo capaz de reduzir $0,465 \mathrm{ca}-$ sos para cada tonelada coletada. As duas variáveis são capazes de explicar mais de $75 \%$ da variação do modelo. Nesse contexto, pode-se afirmar que grande parte da responsabilidade sobre a prevenção dos casos de Dengue, reside sobre o poder púbico e a sua capacidade de coletar de forma contínua e eficiente o lixo da cidade.

Além de ser um potencial criadouro, uma possível explicação da relação entre lixo doméstico e casos de Dengue pode residir no fato de que Aedes aegypti alimenta-se de frutas e vegetais adocicados ${ }^{11}$, algo abundante no lixo residencial. Esse sortimento alimentar pode contribuir para o aumento populacional do mosquito, provendo nutrientes necessários nos diversos estágios de seu desenvolvimento.

Um estudo realizado para avaliar o Aedes aegypti em condições laboratoriais, observou que a alta densidade larvária e a carência de alimentos causaram redução no tamanho de mosquitos adultos e um aumento na taxa de mortalidade ${ }^{29}$. Além disso, a existência de recipientes com lixo próximo às residências está associada a alta incidência de dengue ${ }^{30}$. A ocorrência de dengue onde a coleta é regular, pode apontar que o lixo não teria tempo suficiente para comportar o ciclo reprodutivo, mas seria capaz de alimentar a população dos mosquitos da região.
Em estudo realizado em North Queensland, Austrália, constatou-se que os recipientes com abundância de matéria orgânica tenderam a produzir um mosquito adulto maior e com rápido desenvolvimento ${ }^{31}$. Nesse contexto, é forte a suposição de que o lixo doméstico influencia no volume populacional, não apenas devido à existência de criadouros, mas por oferecer um suprimento alimentar demandado pelo mosquito ao longo de parte seu ciclo de vida.

Já a relação entre casos de dengue e pneus é bem consolidada na literatura ${ }^{32,33}$ pode ter apresentado um valor do coeficiente angular mais elevado em função desse tipo de coleta não obedecer a uma frequência tão regular quanto a coleta de lixo doméstico. Esses dados reforçam a já conhecida necessidade de destinação correta dos pneus e da intensificação da coleta. Essa coleta deve, preferencialmente, apresentar frequência inferior ao ciclo reprodutivo do mosquito.

O Variance Inflation Factor da regressão foi de 1,059 , indicando que o erro padrão é 1,06 vezes maior do que seria se não existe correlação entre as variáveis preditoras (Lixo Doméstimo e Pneus). Isso aponta que existe um baixo impacto da multicolinearidade sobre os valores preditos de Dengue. Porém, é preciso reconhecer que o R quadrado de $75 \%$ é demasiadamente elevado para associar os casos de Dengue a uma única causa (lixo). Fatores como distância dos centros urbanos, renda, clima, distribuição de água, sexo e até mesmo a imunidade natural por aqueles que já foram contaminados são importantes variáveis de confusão que podem ocasionar uma modificação de efeito no coeficiente de determinação.

Podemos destacar que existe uma limitação de dados acerca da característica das coletas de lixo, uma vez que o veículo coletor segue rotas que contemplam bairros considerados mais carentes e ricos, inviabilizando assim uma analise estatística diferencial. Ou seja, em muitas rotas, não é possível segregar com precisão o lixo oriundo de bairros mais carentes e bairros mais ricos.

Um fator relevante que deve ser ressaltado nesse estudo é o fato de que, no período selecionado para análise, existia no Recife a co-circulação de outras arboviroses, como a Zika e a Chikungunya. Aliado a isso, grande parte dos casos foram diagnosticados através de exames clínicos e, por vezes laboratoriais, o que não afasta a possibilidade vieses no diagnóstico, tendo em vista que o quadro clínico dessas arboviroses podem ser confundidos. 


\section{Conclusões}

Com base no modelo, observou-se que as pesagens de lixo apresentaram uma relação inversamente proporcional aos casos de dengue. Também foi possível evidenciar que o aumento nas pesagens, pode proporcionar significativas reduções nos casos notificados da doença. Esse aumento pode ser obtido através de ações como otimização de rotas e aumento das frequências de coleta. É perceptível que isso acarretará um aumento nos custos de coleta urbana, gerando um trade-off entre custos de coleta e os impactos sociais, econômicos e financeiros decorrentes da epidemia de Dengue, Chikungunya e Zika.
Considerando que as pesagens de lixo doméstico foi um bom preditor dos casos de dengue e, que apresentaram uma forte correlação negativa, foi levantada a suposição de que o lixo doméstico poderia não apenas servir como criadouro, mas como fonte de suprimento alimentar para o mosquito. Nesse contexto, para futuros trabalhos, sugerimos que se estude a associação e a reta de regressão entre a coleta seletiva de lixos orgânicos e os casos de dengue, de forma a testar a hipótese de que rejeitos orgânicos presentes no lixo doméstico contribuem para o aumento populacional do Aedes aegypti.

\section{Colaboradores}

Marcos Felipe Falcão Sobral - Concepção do projeto e do estudo, revisão bibliográfica, análise e interpretação dos dados, gráficos, revisão e contribuições críticas na redação do manuscrito. Ana Iza Gomes da Penha Sobral - Levantamento bibliográfico, revisão bibliográfica, redação do artigo, revisão do artigo na versão final. 


\section{Referências}

1. Guzman MG, Halstead SB, Artsob H, Yoksan S, Peeling RW. Dengue: a continuing global threat. Nature Reviews Microbiology 2010; 7:16.

2. Amaku M, Coutinho FA, Massad E. Why dengue and yellow fever coexist in some areas of the world and not in others? Biosystems 2011; 106(2-3):111-120.

3. Gouvêa MM. Time-spatial model on the dynamics of the proliferation of Aedes aegypti. Communications in Nonlinear Science and Numerical Simulation 2017; 44:130-143.

4. Normile D. Surprising new dengue virus throws a spanner in disease control efforts. Science 2013; 342:415.

5. Guzmán MG, Kourí G. Dengue: An update. Lancet Infectious Diseases 2002; 2(1):33-42.

6. Benelli G. Plant-mediated biosynthesis of nanoparticles as an emerging tool against mosquitoes of medical and veterinary importance: a review. Parasitol Res 2016; 115(1):23-34.

7. Benelli G, Mehlhorn H. Declining malaria, rising of dengue and Zika virus: insights for mosquito vector control. Parasitol Res 2016; 115(5):1747-1754.

8. Ebi KL, Nealon J. Dengue in a changing climate. Environ Res 2016; 151:115-123.

9. Bhatt S, Gething PW, Brady OJ, Messina JP, Farlow AW, Moyes CL, Drake JM, Brownstein JS, Hoen AG, Sankoh O, Myers MF, George DB, Jaenisch T, Wint GR, Simmons CP, Scott TW, Farrar JJ, Hay SI. The global distribution and burden of dengue. Nature 2013; 496(7446):504-507.

10. World Health Organization (WHO). Global strategy for dengue prevention and control 2012-2020. Genebra: WHO; 2012.

11. Brasil. Portal da Saúde SUS. SVS Dengue [acessado 2016 Nov 15]. Disponível em: http://portalsaude. saude.gov.br/index.php/o-ministerio/principal/secretarias/svs/dengue.

12. Bohra A, Andrianasolo H. Application of GIS in Modeling of Dengue Risk Based on Sociocultural Data: Case of Jalore, Rajasthan, India. Dengue Bulletin 2001; 25:92-102.

13. Abeyewickreme W, Wickremasinghe AR, Karunatilake K, Sommerfeld J, Axel K. Community mobilization and household level waste management for dengue vector control in Gampaha district of Sri Lanka; an intervention study. Pathogens and Global Health 2012; 106(8):479-487.

14. França RG, Ruaro ECR. Diagnóstico da disposição final dos resíduos sólidos urbanos na região da Associação dos Municípios do Alto Irani (AMAI), Santa Catarina. Cien Saude Colet 2009; 14(6):2191-2197.

15. Associação Brasileira de Empresas de Limpeza Pública e Resíduos Especiais - ABRELPE. Panorama dos Resíduos Sólidos no Brasil. [acesso em 25 ago 2016]. Disponível em: http://www.abrelpe.org.br/Panorama/ panorama2014.pdf.
16. Rodrigues W, Magalhães Filho LNL, Pereira RdS. Análise dos Determinantes dos custos de resíduos sólidos urbanos nas capitais estaduais brasileiras. Urbe. Rev. Bras.Gestão Urb. 2016; 8(1):130-141.

17. Tauil PL. Aspectos críticos do controle da dengue no Brasil. Cad Saude Publica 2002; 18(3):867-871.

18. Souza-Santos R. Fatores associados à ocorrência de formas imaturas de Aedes aegypti na Ilha do Governador, Rio de Janeiro, Brasil. Rev. Soc. Bras. Med. Trop. 1999; 32(4):373-382.

19. Tauil PL. Urbanização e ecologia do dengue. Cad Saude Publica 2001; 17(Supl.):99-102.

20. Zara ALdSA, Santos SM, Fernandes-Oliveira ES, Carvalho RG, Coelho GE. Estratégias de controle do Aedes aegypti: uma revisão. Epidemiol. Serv. Saúde 2016; 25(2):391-404.

21. Lefèvre AMC, Ribeiro AF, Marques GRAM, Serpa LLN, Lefèvre F. Representações sobre dengue, seu vetor e ações de controle por moradores do município de São Sebastião, litoral Norte do Estado de São Paulo, Brasil. Cad Saude Publica 2007; 23(7):1696-1706.

22. Penna MLF. Um desafio para a saúde pública brasileira: o controle do dengue. Cad Saude Publica 2003; 19(1):305-309.

23. Solidum JN, Solidum GG. Correlation of Climate Change Factors with Dengue Incidence in Old Balara, Quezon City, Philippines. IAMURE International Journal of Ecology and Conservation 2016; 17:113-122.

24. Costa AIP, Natal D. Distribuição espacial da dengue e determinantes socioeconômicos em localidade urbana no Sudeste do Brasil. Rev Saude Publica 1998; 32(3):232-236.

25. Honorato T, Lapa PPA, Sales CMM, Reis-Santos B, Tristão-Sá R, Bertolde AI, Maciel EL. Spatial analysis of distribution of dengue cases in Espírito Santo, Brazil, in 2010: use of Bayesian model. Rev. Brasil. Epidemiol. 2014; 17(2):150-159.

26. Flauzino RF, Souza-Santos R, Oliveira RMd. Indicadores Socioambientais para Vigilância da Dengue em Nível Local. Saúde Soc. 2011; 20(1):225-240.

27. Medronho RA, Macrini L, Novellino DM, Lagrotta MTF, Câmara VM, Pedreira CE. Aedes aegypti Immature Forms Distribution According to Type of Breeding Site. Am. J. Trop. Med. Hyg. 2009; 80(3):401-404.

28. Dom NC, Madzlan MF, Yusof SNN, Ahmad AH, Ismail R, Camalxaman SN. Profile distribution of juvenile Aedes species in an urban area of Malaysia. Trans $R$ Soc Trop Med Hyg 2016; 110(4):237-245.

29. ECORECIFE. Calendário de Coleta [acessado 2016 Ago 21]. Disponível em: http://www.ecorecife.org/coleta/ domiciliar.

30. Gama RA, Alves KdC, Martins RF, Eiras AE, Resente MC. Efeito da densidade larval no tamanho de adultos de Aedes aegypti criados em condições de laboratório. Revi. Soc. Brasil. Med. Trop. 2005; 38(1):64-66. 
31. Thammapalo S, chongsuvivatwong V, geater A, Dueravee $M$. Environmental factors and incidence of dengue fever and dengue haemorrhagic fever in an urban area, Southern Thailand. Epidemiol. Infect. 2008; 136(1):135143.

32. Tun-Lin W, Burkot TR, Kay BH. Effects of temperature and larval diet on development rates and survival of the dengue vector Aedes aegypti in north Queensland, Australia. Med. Vet. Entomol. 2000; 14(1):31-37.

33. Lounibos LP. Invasions by insect vectors of human disease. Annu. Rev. Entomol 2002; 47:233-266.

Artigo apresentado em 30/08/2016

Aprovado em 22/05/2017

Versão final apresentada em 24/05/2017 